\title{
On the size of prime factors of integers
}

by

\author{
J. D. BoveX (Cardiff)
}

1. Introduction. For $n$ a positive integer and $y>1$ a real number we define

$$
d(y, n)=\max \{d|d| n ; p \mid d \text { and } p \text { prime } \Rightarrow p<y\}
$$

and $\gamma(y, n)=(\log d(y, n)) / \log y$.

In this paper we make more precise some results of Erdös [4] on the size of $\gamma(p, n)$ where $p$ is a prime factor of $n$. For real $u \geqslant 0$ define

$$
p(u, n)=\sum_{\substack{p \mid n \\ \gamma(p, n)>u}} 1,
$$

we then have the following "Turán's method" result:

THEOREM 1. For $\varphi(u, n)$ as defined above and $\tau(u)$ defined below we have

$\sum_{n \leqslant x} \varphi(u, n)=x(1+o(1)) \tau(u) \log _{2} x+o(x)$
(ii) $\quad \sum_{n \leqslant x}\left(\varphi(u, n)-\tau(u) \log _{2} n\right)^{2}=x\left\{\left(\left(\left(\tau(u) \log _{2} x\right)^{2}\right)+o\left(1+\tau(u) \log _{2} x\right)\right\}\right.$

uniformly in $u$ as $x \rightarrow \infty$.

Here and elsewhere in this paper $\log _{k} n$ denotes the $k$-fold iterated logarithm.

The function $\tau(u)$ is defined as follows. Let $\varrho(u)$ be the real valued function defined by the following properties

(1) $\left\{\begin{array}{l}\varrho(u)=0(u<0) ; \varrho(u)=1(0 \leqslant u \leqslant 1), \\ u \varrho^{\prime}(u)=-\varrho(u-1)(u>1) ; \varrho(u) \text { is continuous for } u>0 .\end{array}\right.$

De Bruijn [3] has studied the asymptotic behaviour of $\varrho(u)$ in some detail and in particular has shown that
(2)
$\varrho(u)=\exp \left\{-u \log u-u \log _{2} u+O(u)\right\}$
as $u \rightarrow \infty$. 
We define

$$
\tau(u)=e^{-\gamma} \int_{u}^{\infty} \varrho(v) d v .
$$

For large $u, \tau(u)$ behaves very like $\varrho(u)$, in fact we have

$$
\tau(u)=\left(e^{-\gamma}+o(1)\right)(u+1) \varrho(u+1) \quad \text { as } u \rightarrow \infty .
$$

A proof of (3) goes as follows

$$
\begin{aligned}
\tau(u) & =e^{-\gamma} \int_{u+1}^{\infty} \varrho(v-1) d v \\
& =-e^{-\gamma} \int_{u+1}^{\infty} v \varrho^{\prime}(v) d v \quad \text { by }(1) \\
& =-e^{-\gamma}[v \varrho(v)]_{u+1}^{\infty}+e^{-\gamma} \int_{u+1}^{\infty} \varrho(v) d v
\end{aligned}
$$

which gives

$$
\tau(u)-\tau(u+1)=e^{-\gamma}(u+1) \varrho(u+1) .
$$

Summing we get

$$
\tau(u)=e^{-\gamma}(u+1) \varrho(u+1)+e^{-\gamma} \sum_{k=2}^{\infty}(u+k) \varrho(u+k) .
$$

It can easily be verified from De Bruijn's asymptotic formula for $\varrho(u)$ [3] that $\varrho(u+1) \ll \varrho(u) / u$ and (3) follows.

$$
\text { Put }
$$

$$
P(n)=\max _{p \mid n} \gamma(p, n) .
$$

Erdös [4] has shown that for almost all $n$ (i.e. on a sequence with asymptotie density 1)

$$
\text { (4) } \quad P(n)=(1+o(1)) \log _{3} n / \log _{4} n \text {. }
$$

Using Theorem 1 we can obtain a more precise result than this. For $x>e^{e}$ we define $\xi(x)$ to be the root of

$$
\tau(\xi) \log _{2} x=1 .
$$

THEOREM 2. For almost all integers $n$

$$
P(n)=\xi(n)+o(1) .
$$

Proof. It follows from (2) and (3) that for any $\varepsilon>0$

$$
\tau(\xi(x)+\varepsilon) \log _{2} x \rightarrow 0 \text { and } \tau(\xi(x)-\varepsilon) \log _{2} x \rightarrow \infty \text { as } x \rightarrow \infty
$$

but this, combined with the slow rate of growth of $\xi(n)$ and with Theorem 1 , implies that for almost all $n$

$$
\varphi(\xi(n)+\varepsilon, n)=0 \quad \text { and } \quad \varphi(\xi(n)-\varepsilon, n) \rightarrow \infty
$$

and the result follows.

In the same paper Erdös outlined a proof that there exists a continuous function $\varphi(u)$ such that for fixed $u$ and almost all $n \varphi(u, n)$ $=(1+o(1)) \varphi(u) \log _{2} n$. It follows at once from Theorem 1 that this result holds with $\varphi(u)=\tau(u)$ and in fact we have

THEOREM 3. For almost all integers $n$

$$
\sup _{u \geqslant 0}\left|\frac{\varphi(u, n)}{\log _{2} n}-\tau(u)\right| \rightarrow 0 .
$$

Proof. Let $\varepsilon>0$, it is enough to show that for almost all $n$ and all $u \geqslant 0$

$$
\left|\varphi(u, n) / \log _{2} n-\tau(u)\right|<\varepsilon .
$$

Choose an integer $N>0$ and ar real number $A>0$ to satisfy $\tau(A)<\varepsilon / 4$ and $A / N<\varepsilon / 2$. By Theorem 1 (i)

$$
\sum_{n \leqslant x} \varphi(A, n) / \log _{2} n \leqslant \tau(A) x+o(x) \leqslant(\varepsilon / 3) x \quad \text { for large } x,
$$

and so we can certainly say that for almost all $n$ and for $u \geqslant A$

$$
|\varphi(u, n)| \log _{2} n-\tau(u) \mid<\varepsilon .
$$

Next, by Theorem 1 (ii), we can say that for almost all $n$ and for integer $k$ with $0 \leqslant k \leqslant N$

$$
\left|\frac{\varphi(k A \mid N, n)}{\log _{2} n}-\tau\left(\frac{k A}{N}\right)\right|<\frac{\varepsilon}{2} .
$$

For $0 \leqslant u<A$ there is some $k$ such that $k A / N \leqslant u<(k+1) A / N$ and we have

$$
\left|\varphi(u, n) / \log _{2} n-\tau(u)\right|<\frac{\varepsilon}{2}+\tau(k A / N)-\tau((k+1) A \mid N)<\frac{\varepsilon}{2}+\frac{A}{N} \leqslant \varepsilon .
$$

We can also determine the average value of $\gamma(p, n)$ for almost all $n$

$$
\frac{1}{\log _{2} n} \sum_{p \mid n} \gamma(p, n)=-\frac{1}{\log _{2} n} \int_{0}^{\infty} u d \varphi(u, n)
$$

integrating by parts

$$
=\int_{0}^{\infty} \frac{\varphi(u, n)}{\log _{2} n} d u
$$


and it is not hard to show using Theorem 3 and Theorem 1 (i) that for almost all $n$

$$
\int_{0}^{\infty} \frac{\varphi(u, n)}{\log _{2} n} d u=(1+o(1)) \int_{0}^{\infty} \tau(u) d u=(1+o(1)) f^{\prime}(0)=1+o(1),
$$

with $f(z)$ as defined in $\$ 2$. Hence for almost all integers $n$

$$
\frac{1}{\log _{2} n} \sum_{p \mid n} \gamma(p, n)=(1+o(1)) \text {. }
$$

If $y>w \geqslant 0$ are real numbers we use the notation $\sum_{n}^{w, y} a(n)$ to denote the sum of $a(n)$ only over $n$ with no prime factors $<w$ or $\geqslant y$. We abbreviate $\sum^{0, y}$ by $\Sigma^{y}$. To prove Theorom 1 we have to estimate sums of the form

$$
\sum_{a>y^{u}}^{w, y} \frac{1}{a}
$$

This could probably be done using the methods of de Bruijn ([1] and [2]), but instead we use a different method using Fourier transforms and a result borrowed from probability theory (Lemma 3 ). This has the advantage of being fairly self contained and also enables us to get better error terms than I was able to get using de Bruijn's methods. In $\S 2$ we prove some lemmas and Theorem 1 (i), then in $\S 3$ we complete the proof of Theorem 1.

2. For real $u$ and real $y>0$ we define

$$
F_{y}(u)=\prod_{p<y}\left(1-\frac{1}{p}\right) \sum_{a \leqslant y^{u}} \frac{1}{a},
$$

then for each $y F_{y}(u)$ is a distribution function with support in $(0, \infty)$. For complex $z$ with $\operatorname{Re} z<\log y$ we define

$$
f_{y}(z)=\int_{0}^{\infty} e^{u z} d F_{y}(u)
$$

If we write the integral as a sum we see that

$$
f_{y}(z)=\prod_{p<y}\left(1-\frac{1}{p}\right)\left(1-\frac{1}{p^{1-z / \log y}}\right)^{-1}
$$

and so $f_{y}(z)$ is analytic for $\operatorname{Re} z<\log y$.

Finally we define

$$
f(z)=e^{-\gamma} \int_{0}^{\infty} e^{u z} \varrho(u) d u
$$

\section{LemMa 1.}

$$
f(z)=\exp \left\{\int_{0}^{\tilde{z}} \frac{e^{s}-1}{s} d s\right\}
$$

\section{Proof.}

$$
\begin{aligned}
f(z) & =e^{-\gamma} \int_{0}^{\infty} e^{(u-1) \varepsilon} \varrho(u-1) d u \\
& =-e^{-\gamma} e^{-z} \int_{1}^{\infty} e^{u z} u \varrho^{\prime}(u) d u \quad \text { by }(1) \\
& =-e^{-\gamma} e^{-z} \frac{d}{d z} \int_{0}^{\infty} e^{u z} \varrho^{\prime}(u) d u \text { as } \varrho^{\prime}(u)=0 \text { for } 0<u<1 \\
& =-e^{-\gamma} e^{-z} \frac{d}{d z}\left\{\left[e^{u \varepsilon} \varrho(u)\right]_{0}^{\infty}-z \int_{0}^{\infty} e^{u z} \varrho(u) d u\right\} \\
& =e^{-z}\left(s f^{\prime}(z)+f(z)\right),
\end{aligned}
$$

i.e.

$$
\frac{f^{\prime}(z)}{f(z)}=\frac{e^{z}-1}{z}
$$

Hence

$$
f(z)=\exp \left\{\int_{0}^{z} \frac{e^{s}-1}{s} d s\right\}=c g(z) \text { say. }
$$

Integrating (6) by parts gives

$$
z c g(z)=z f(z)=e^{-\gamma}\left[e^{z u} \varrho(u)\right]_{0}^{\infty}-e^{-\gamma} \int_{0}^{\infty} e^{s u} \varrho^{\prime}(u) d u .
$$

If we put $z=i t$ and let $t \rightarrow \infty$ the integral on the right tends to 0 and we get

$$
e^{-\gamma}=c \lim _{t \rightarrow \infty} i \operatorname{tg}(i t)=c e^{-\gamma} \quad(\text { see }[3] \text { for example) }
$$

and so $e=1$

If we put $z=0$ in (6) we see that

$$
e^{-\gamma} \int_{0}^{\infty} \varrho(u) d u=1
$$

and so if we define $F(u)=e^{-r} \int_{-\infty}^{u} \varrho(w) d w$ then $F(u)$ is a distribution function. 
LFinMa 2. For any complex number $z$

$$
\lim _{y \rightarrow+\infty} f_{\nu}(z)=f(z)
$$

In addition for real t and integer $y \geqslant 3$ with $0 \leqslant t \leqslant \log y$

$$
\begin{aligned}
\left|\frac{f_{y}(1+i t)}{f_{y}(1)}-\frac{f(1+i t)}{f(1)}\right| & =O\left(\log ^{-1} y\right) \frac{t}{t+1} \\
\left|f_{y}(1)-f(1)\right| & =O\left(\log ^{-1} y\right) .
\end{aligned}
$$

Proof. Let $\sigma=\operatorname{Re} z$ and $\log y>2 \sigma$. We will show that

$$
\frac{f_{y}^{\prime}(z)}{f_{y}(z)}=\frac{e^{z}-1}{z}+O\left(\frac{e^{\sigma}}{\log y}\right)
$$

If we integrate (7) and observe that $f_{y}(0)=f(0)=1$ we see that $\log f_{y}(z)$ $\rightarrow \log f(z)$ as $y \rightarrow \infty$ and (i) follows. The proofs of (ii), (iii) and (iv) are very similar and so we just prove (iii). Write

then

$$
g_{y}(t)=\frac{f_{u}(1+i t) f(1)}{f(1+i t) f_{y}(1)}
$$

$$
\frac{g_{y}^{\prime}(t)}{g_{y}(t)}=\frac{1}{i}\left(\frac{f_{y}^{\prime}(1+i t)}{f_{y}(1+i t)}-\frac{f^{\prime}(1+i t)}{f(1+i t)}\right)=O\left(\log ^{-1} y\right) \quad \text { by }(\tau)
$$

If we put

$$
G_{y}(t)=\int_{0}^{t} \frac{g_{y}^{\prime}(x)}{g_{y}(x)} d x
$$

then

Hence

$$
G_{y}(t)=O\left(t \log ^{-1} y\right) \quad \text { and } \quad g_{y}(t)=\exp G_{y}(t)
$$

$$
g_{y}(t)-1=G_{y}(t)\left\{\left(\exp G_{y}(t)-1\right) / G_{y}(t)\right\}=O\left(t \log ^{-1} y\right)
$$

for $t \leqslant \log y$. Moltiplying by $f(1+i t) / f(1)$ we get

$$
\frac{f_{y}(1+i t)}{f_{y}(1)}-\frac{f(1+i t)}{f(1)}=t f(1+i t) O\left(\log ^{-1} y\right)
$$

and (iii) follows as t $(1+i t)$ is easily shown to be bounded.
We now prove $(7)$

$$
\begin{aligned}
\frac{f_{y}^{\prime}(z)}{f_{y}(z)} & =\sum_{p<y} \frac{\log p}{\log y} p^{(z / \log y)-1}\left(1-p^{(z / \log y)-1}\right)^{-1} \\
& =\frac{1}{\log y} \sum_{p<y} p^{(z / \log y)-1} \log p+o\left(\frac{1}{\log y} \sum_{p<y} \frac{\log p}{p^{2(1-\sigma / \log y)}}\right) \\
& =\frac{1}{\log y} \sum_{p<y} p^{(z / \log y)-1} \log p+O\left(\frac{e^{\sigma}}{\log y}\right)
\end{aligned}
$$

as the sum in brackets is convergent for $2 \sigma<\log y$. If we write the sum above as an integral we get

$$
\begin{array}{r}
\frac{f_{y}^{\prime}(z)}{f_{y}(z)}=\frac{1}{\log y} \int_{1}^{y} x^{(z / \log y)-1} \log x \cdot d[\operatorname{li} x]+\frac{1}{\log y} \int_{1}^{y} x^{(z / \log y)-1} \log x \cdot d[\pi(x)-\operatorname{li} x]+ \\
+o\left(\frac{e^{\sigma}}{\log y}\right)
\end{array}
$$

where li $x$ is the logarithmic integral

$$
\lim _{\delta \rightarrow 0}\left(\int_{0}^{1-\delta} \frac{1}{\log t} d t+\int_{1+\delta}^{x} \frac{1}{\log t} d t\right)
$$

and $\pi(x)$ is the number of primes $\leqslant x$. The first integral above is equal to

$$
\frac{1}{\log y} \int_{1}^{y} x^{(\theta / \log y)-1} d x=\frac{e^{z}-1}{z}
$$

as required.

If the second integral is integrated by parts it equals

$$
\begin{gathered}
\frac{1}{\log y}\left[x^{(z / \log y)-1} \log x(\pi(x)-\operatorname{li} x)\right]_{1}^{y}-\frac{1}{\log y} \int_{1}^{y}(\pi(x)-\operatorname{li} x) x^{(z / \log y)-2} O(\log x) d x \\
\ll \frac{e^{\sigma}}{\log y}\left\{\frac{\log y}{y}(\pi(y)-\operatorname{li} y)+\int_{1}^{y}(\pi(x)-\operatorname{li} x) \frac{\log x}{x^{2}} d x\right\}
\end{gathered}
$$

but by the prime number theorem $|\pi(x)-\operatorname{li} x| \ll \frac{x}{(\log x)^{2}}$ and the result follows. 
The next lemma is due to Esseen ([5], Theorem 2a). We have replaced the condition $\left|G^{\prime}(u)\right| \leqslant M I$ by $(G(u)-G(v)) /(u-v) \leqslant M$ but the proof is the same.

LEMve 3. If $G(u)$ and $H(u)$ are two distribution functions,

$$
g(t)=\int_{-\infty}^{\infty} e^{i u t} d G(u), \quad h(t)=\int_{-\infty}^{\infty} e^{i u t} d H(u)
$$

and $(G(u)-G(v)) /(u-v) \leqslant M$ for all $u$ and $v$ then

$$
|G(u)-H(u)| \ll \int_{0}^{T} \frac{|g(t)-h(t)|}{t} d t+\frac{M}{T}
$$

for all $u$ and all $T>0$.

We are now ready to obtain our estimate for $F_{v}(u)$.

LexcMa 4. For integer $y \geqslant 2$ and real $u \geqslant 0$

$$
\prod_{p<y}\left(1-\frac{1}{p}\right) \sum_{a>y^{u}} \frac{1}{a}=\tau(u)+o\left(\frac{e^{-u}\left|\log _{2} y\right|}{\log y}\right) .
$$

Proof. For $y=2$ and $u>0$ the left hand side is zero but the error term is greater than the main term. For $y \geqslant 3$ write

$$
\begin{aligned}
& c_{y}=\int_{0}^{\infty} e^{v} d F_{y}(v)=f_{y}(1), \quad c=\int_{0}^{\infty} e^{v} d F(v)=f(1), \\
& G_{y}(u)=\int_{-\infty}^{u} e^{v} d F_{y}(v) \quad \text { and } \quad G(u)=\int_{-\infty}^{u} e^{v} d F(v) .
\end{aligned}
$$

Then $c_{y}^{-1} G_{y}(u)$ and $c^{-1} G(u)$ are distribution functions and

$$
\int_{0}^{\infty} e^{i u t} d\left(c_{y}^{-1} G_{y}(u)\right)=c_{y}^{-1} \int_{0}^{\infty} e^{u(1+i t)} d F_{y}(u)=\frac{f_{y}(1+i t)}{f_{y}(1)},
$$

similarly

$$
\int_{0}^{\infty} e^{i t} d\left(c^{-1} G(u)\right)=\frac{f(1+i t)}{f(1)}
$$

$G(u)$ clearly satisfies the condition of Lemma 3 with $M=\sup e^{u} \varrho(u)$, and so by Lemma 2 (iii) and Lemma 3

$$
\left|c_{y}^{-1} G_{y}(u)-c^{-1} G(u)\right| \ll \frac{1}{\log y} \int_{0}^{\log y} \frac{d t}{t+1}+\frac{1}{\log y} \ll \frac{\log _{2} y}{\log y} .
$$

Lemma 2 (iv) implies that $e_{y}^{-1}=c+O\left(\log ^{-1} y\right)$ and so, for $y \geqslant 3$ and $u \geqslant 0$,

Now

$$
G_{y}(u)=G(u)+O\left(\log _{2} y / \log y\right)
$$

$$
\prod_{p<y}\left(1-\frac{1}{p}\right) \sum_{a>y^{u}} \frac{1}{a}=\int_{u}^{\infty} d F_{y}(v)=\int_{u}^{\infty} e^{-v} d G_{y}(v)
$$

integrating by parts

$$
\begin{aligned}
& =\left[e^{v} G_{y}(v)\right]_{u}^{\infty}+\int_{u}^{\infty} e^{-v} G_{y}(v) d v \\
& =\left[e^{-v} G(v)\right]_{u}^{\infty}+\int_{u}^{\infty} e^{-v} G(v) d v+O\left(e^{-u} \log _{2} y / \log y\right) \quad \text { by }(8) \\
& =\int_{u}^{\infty} e^{-v} d G(v)+O\left(e^{-u} \log _{2} y / \log y\right)=\tau(u)+O\left(e^{-u} \log _{2} y / \log y\right)
\end{aligned}
$$

as required.

Our final lemma in this section is due to de Bruijn [1].

Leanara 5. Let $\Phi(x, y)$ denote the number of integers $\leqslant x$ all of whose prime factors are $\geqslant y$, then

$$
\Phi(x, y)-1=x \prod_{p<y}\left(1-\frac{1}{p}\right) \psi(x, y)
$$

where

$$
\psi\left(y^{u}, y\right)=1+O\left(e^{-u \alpha}\right) \quad \text { for } \quad y \geqslant 2, u \geqslant 0
$$

and $a$ an absolute positive constant.

Proof. For $u \geqslant 1$ this follows at once from [1], 1.16. For $0 \leqslant u<1$ the result is trivial as $\Phi(x, y)=1$ if $x<y$.

We are now ready to prove Theorem 1 (i). If we write

then

$$
\delta(u, p, n)= \begin{cases}1 & \text { if } p \mid n \text { and } \gamma(p, n)>u \\ 0 & \text { otherwise }\end{cases}
$$

$\sum_{n \leqslant x} \varphi(u, n)=\sum_{n \leqslant x} \sum_{p \leqslant x} \delta(u, p, n)=\sum_{p \leqslant x} \sum_{n \leqslant x} \delta(u, p, n)=\sum_{x \leqslant x} \sum_{a>p^{u}}^{p} \Phi\left(\frac{x}{a p}, p\right)$ 


$$
=\sum_{p \leqslant x} \sum_{a>p^{u}}^{p}\left(\Phi\left(\frac{x}{a p}, p\right)-1\right)+N(u, x)
$$

say, where $N(u, x)$ is the number of $n \leqslant x$ whose largest prime factor is $<n^{1 /(1+u)}$. It is not hard to see that

(9) $\quad N(u, x)=\left\{\begin{array}{lll}o(x) & \text { if } & u \geqslant \log _{4} x \\ o(x)=o\left(\chi \tau(u) \log _{2} x\right) & \text { if } & u \leqslant \log _{4} x .\end{array}\right.$

Applying Lemma 5 we get

$$
\sum_{n \leqslant x} \varphi(u, n)=x \sum_{p<x} \frac{1}{p} \prod_{p^{\prime}<p}\left(1-\frac{1}{p}\right) \sum_{a>p^{u}}^{p} \frac{1}{a} \psi\left(\frac{x}{a p}, p\right)+N(u, \infty) .
$$

If we abreriate $\sum_{p^{\prime}<p}\left(1-\frac{1}{p^{\prime}}\right)$ by $\pi_{p}$ we can write

(10) $\quad \sum_{n \leqslant x} \varphi(u, n)=x S_{1}+x S_{2}+N(u, x)$

where

$$
S_{1}=\sum_{p \leqslant x} \frac{1}{p} \pi_{p} \sum_{a>p^{u}}^{p} \frac{1}{a} \quad \text { and } \quad S_{2}=\sum_{p \leqslant x} \frac{1}{p} \pi_{p} \sum_{a>p^{u}}^{p} \frac{1}{a}\left(\psi\left(\frac{x}{a p}\right)-1\right)
$$

By Lemma 4

(11) $S_{1}=\tau(u) \sum_{p \leqslant x} \frac{1}{p}+O(1) e^{-u} \sum_{p \leqslant x} \frac{\log _{2} p}{p \log p}=(1+o(1)) \tau(u) \log _{2} x+O\left(e^{-u}\right)$.

If we consider separately the cases $u \geqslant \log _{4} x$ and $u \leqslant \log _{4} x$ we see at once that

$$
e^{-u}=o(1)+o\left(\tau(u) \log _{2} x\right)
$$

We now deal with $S_{2} \cdot \psi(x, y)$ is bounded for all $x, y$ and so we have

$$
S_{2} \ll S_{3}+S_{4}+S_{5}
$$

where, writing $x^{\prime}=x^{1 /\left(u+1+2 \log _{2} x\right)}$,

$$
S_{3}=\sum_{x^{\prime} \leqslant p \leqslant x} \frac{1}{p} \pi_{p} \sum_{a>p^{u}}^{p} \frac{1}{a}, \quad S_{4}=\sum_{p \leqslant x} \frac{1}{p} \pi_{p} \sum_{a>p^{u+\log _{2} x}}^{p} \frac{1}{a}
$$

and

$$
S_{5}=\sum_{p<x^{\prime}} \frac{1}{p} \pi_{p} \sum_{p^{u}<a \leqslant p^{u+1 \log _{2} x}}^{p} \frac{1}{a} e^{-a \log _{2} x}
$$

$S_{5} \ll \log _{2} x /(\log x)^{\alpha}=o(1)$ and it follows from Lemma 4 that $S_{4}$ $\ll \log _{2} x / \log x=o(1)$. Also by Lemma 4

$$
S_{3} \ll \tau(u) \log \left(u+1+2 \log _{2} x\right)+\sum_{x^{\prime} \leqslant p<x} \frac{\log _{2} p}{p \log p}=\tau(u) \circ\left(\log _{2} x\right)+o(1)
$$

as required.

We have shown that $S_{2}=o\left(\tau(u) \log _{9} x\right)+o(1)$ and this in conjunction with (9), (10), (11) and (12) completes the proof of Theorem 1 (i).

3. Theorem 1 (ii). Before we can complete the proof of Theorem 1 we need two more lemmas.

LEMma 6. For integer $y$, with $y>v \geqslant 2$ and for all $u \geqslant 0$ we have

$$
\prod_{w \leqslant p<y}\left(1-\frac{1}{p}\right) \sum_{a>y^{u}}^{w^{u}, y} \frac{1}{a}=\tau(u)+O\left(\frac{\log (v+1)}{v}\right)
$$

with $v=(\log y) / \log w$.

Proof. If we define

$$
F_{w, y}(u)=\prod_{u \leqslant p<y}\left(1-\frac{1}{p}\right) \sum_{a \leqslant u^{u}}^{w, y} \frac{1}{a}
$$

then $F_{v, y}(u)$ is a distribution function with characteristic function $f_{y}(i t) \mid f_{w}(i t / v)$. In the light of Lemma 3 it will be enough to show that

$$
\int_{0}^{v}\left|\frac{f_{y}(i t)}{f_{w}(i t / v)}-f(i t)\right| \frac{d t}{t}=o\left(\frac{\log (v+1)}{v}\right) .
$$

Clearly $f_{w}(i t / v)=1+O(t / v)$ and $f_{w}(i t / v)^{-1}=O(1)$ for $t \leqslant v$ and all $z$. Hence

$$
\begin{aligned}
\int_{0}^{v}\left|\frac{f_{y}(i t)}{f_{w}(i t / v)}-f(i t)\right| \frac{d t}{t} & \ll \int_{0}^{v}\left|f_{y}(i t)-f_{w}(i t / v) f(i t)\right| \frac{d t}{t} \\
& \ll \int_{0}^{v}\left|f_{y}(i t)-f(i t)\right| \frac{d t}{t}+\frac{1}{v} \int_{0}^{v}|f(i t) t| \frac{d t}{t+1} \\
& \ll \frac{1}{v} \int_{0}^{v} \frac{d t}{t+1} \ll \frac{\log (v+1)}{v}
\end{aligned}
$$

by Lemma 2 (ii) and because $t f(i t)$ is bounded. 
LEMMA 7 . For all real $u, w$ with $w>u>0$ we have

$$
\sum_{k=1}^{\infty} \frac{1}{k}\left\{F(u)-F\left(u-\frac{w}{k}\right)\right\}=o\left(\log \frac{w}{u}\right)+O(1) .
$$

Proof.

$$
\begin{aligned}
\sum_{k=1}^{\infty} \frac{1}{k}\left\{F(u)-F\left(u-\frac{w}{k}\right)\right\} & =\int_{1}^{\infty} \frac{1}{x}\left\{F(u)-F\left(u-\frac{w}{x}\right)\right\} d x+O(1) \\
& =\int_{i}^{\infty} \int_{u-w / x}^{u} \frac{\varrho(v)}{x} d v d x+O(1)
\end{aligned}
$$

changing the order of integration

$$
\begin{aligned}
& =\int_{0}^{u} \varrho(v) \int_{1}^{w /(u-v)} x^{-1} d x d v+O(1) \\
& =\int_{0}^{u} \log \left(\frac{w}{u-v}\right) \varrho(v) d v+O(1) \\
& =\int_{0}^{u} \log \frac{w}{v} \varrho(u-v) d v+O(1) \\
& \ll e^{-u} \int_{0}^{v} \log \frac{w}{v} e^{v} d v+e^{-u} \int_{1}^{u} \log \frac{w}{v} e^{v} d v+O(1)
\end{aligned}
$$

using the fact that $\varrho(v) \ll e^{-v}$ and $\log \frac{w}{v} \geqslant 0$. The first integral above is $\ll e^{-u} \log w+O(1)$

$$
=e^{-u} \log \frac{w}{u}+e^{-u} \log u+O(1) \ll \log \frac{w}{u}+O(1)
$$

as required. Integrating the second integral by parts we get

$$
e^{-u}\left[\log \frac{w}{v} e^{v}\right]_{1}^{u}+e^{-u} \int_{1}^{u} \frac{1}{v} e^{v} d v=\log \frac{w}{v}-e^{-u} \log w+O(1) \ll \log \frac{w}{u}+O(1)
$$

as required.

The proof of Theorem 1 will follow easily if we prove that

$$
\sum_{n \leqslant x} \varphi(u, n)^{2}=x\left\{(1+o(1))\left(\tau(u) \log _{2} x\right)^{2}+O\left(\tau(u) \log _{2} x\right)+O(1)\right\} .
$$

Now

$$
\begin{aligned}
\sum_{n \leqslant x} \varphi(u, n)^{2} & =\sum_{n \leqslant x} \sum_{p \leqslant x} \sum_{q \leqslant x} \delta(u, p, n) \delta(u, q, n) \\
& =2 \sum_{p<q \leqslant x} \sum_{n \leqslant x} \delta(u, p, n) \delta(u, q, n)+\sum_{n \leqslant x} \varphi(u, n) \\
& =2 \sum_{p<q \leqslant x} \sum_{a>p^{u}} \sum_{b>q^{u l a p}}^{p, q} \Phi\left(\frac{x}{a b p q}, q\right)+x O\left(\tau(u) \log _{2} x+1\right) \\
& =2 x \sum_{p<q \leqslant x} \frac{1}{p q} \pi_{p} \sum_{a>p^{u}}^{p} \frac{1}{a} \pi_{q} \mid \pi_{p} \sum_{b>q^{u l a p}}^{\sum^{p, q}} \frac{1}{b} \psi\left(\frac{x}{a b p q}, q\right)+E,
\end{aligned}
$$

where $E$ denotes a function of $x$ and $u$ bounded by the error term in (13). First we deal with the term arising from the error term in Lemma 6 , and show that

(15) $\sum_{p<q \leqslant x} \frac{1}{p q} \pi_{p} \sum_{a>p^{u}}^{p} \frac{1}{a} \log \left(\frac{\log q}{\log p}+1\right) \frac{\log p}{\log q}=O\left(\tau(u) \log _{2} x\right)+O(1)$.

The sum above is clearly bounded by

$$
\begin{aligned}
\sum_{p \leqslant x} \frac{1}{p} \pi_{p} \sum_{a>p^{u}} \frac{1}{a} \sum_{1 \leqslant v \leqslant \log x / \log p} \frac{\log (v+1)}{v} \sum_{p^{v}<q \leqslant p^{v+1}} \frac{1}{q} \\
\ll \sum_{p \leqslant x} \frac{1}{p} \pi_{p} \sum_{a>p^{u}}^{p} \frac{1}{a} \sum_{v=1}^{\infty} \frac{\log (v+1)}{v^{2}}=O\left(\tau(u) \log _{2} x+1\right)
\end{aligned}
$$

as in the proof of part (i).

We next deal with the sum where $q^{u} / a p<b \leqslant q^{u}$. Because of Lemma 6 and (15) we can replace

$$
\pi_{q} / \pi_{p} \sum_{q^{u} / a p<b \leqslant q^{u}}^{\urcorner^{u}, q} \frac{1}{b} \text { by } \quad F\left(u-\frac{\log a}{\log q}-\frac{\log p}{\log q}\right)-F(u),
$$

and so it is enough to get a bound for

$$
\sum_{p<q \leqslant x} \frac{1}{p q} \pi_{p} \sum_{a<p^{u}}^{p} \frac{1}{a}\left\{F^{p}\left(u-\frac{\log a}{\log q}-\frac{\log p}{\log q}\right)-F(u)\right\} .
$$


If we replace the sum over $a$ by an integral and take the sum over $q$ inside we get

$$
\begin{aligned}
\sum_{p<x} \frac{1}{p} \int_{u}^{\infty} \sum_{p<q \leqslant x}\left\{F^{F}\left(u-(w+1) \frac{\log p}{\log q}\right)-F^{\prime}(u)\right\} d F_{p}(w) \\
\quad \leqslant \sum_{p<x} \frac{1}{p} \int_{u}^{\infty} \sum_{k=1}^{\log x} \sum_{p^{k}<q \leqslant p^{k+1}} \frac{1}{q}\left\{F\left(u-\frac{w+1}{k}\right)-F(u)\right\} d F_{p}(w) \\
\ll \sum_{p<x} \frac{1}{p} \int_{u}^{\infty} \sum_{k=1}^{\infty} \frac{1}{k}\left\{F\left(u-\frac{w+1}{k}\right)-F(u)\right\} d F_{p}(w) \\
\ll \sum_{p<x} \frac{1}{p} \int_{u}^{\infty} \log \frac{w+1}{u} d F_{p}(w)+\sum_{p<x} \frac{1}{p} \int_{u}^{\infty} d F_{p}(w)
\end{aligned}
$$

by Lemma 7 . The second term above is just

$$
\sum_{p<x} \frac{1}{p} \pi_{p} \sum_{a>p^{u}}^{p} \frac{1}{a}=O\left(\tau(u) \log _{2} x+1\right) .
$$

If $G_{p}(w)$ is defined as in the proof of Lemma 4 then the first term above equals

$$
\sum_{p<x} \frac{1}{p} \int_{u}^{\infty} \log \frac{w+1}{u} e^{-w} d G_{p}(w)
$$

If we integrate by parts, apply (8), and then reverse the partial integration we find that (16) equals

$$
\begin{aligned}
\sum_{p<x} \frac{1}{p} \int_{u}^{\infty} \log \frac{w+1}{u} e^{-w} d G(w) & +O\left(\sum_{p<x} \frac{\log _{2} p}{p \log p}\right) \\
& =\log _{2} x \int_{u}^{\infty} \log \frac{w+1}{u} d E^{\prime}(w)+O(1)
\end{aligned}
$$

Now

$$
\begin{aligned}
& \int_{u}^{\infty} \log \frac{w+1}{u} d F(w)=-\int_{u}^{\infty} \log \frac{w+1}{u} d \tau(w) \\
& \quad=-\left[\log \frac{w+1}{u} \tau(u)\right]_{u}^{\infty}+\int_{u}^{\infty} \frac{\tau(w)}{w+1} d w \ll \frac{1}{u} \tau(u)+\int_{u}^{\infty} \varrho(w+1) \quad \text { by }(3) \\
& \quad \ll \tau(u)
\end{aligned}
$$

as required.
We are now left with

$$
2 x \sum_{p<q \leqslant x} \frac{1}{p q} \pi_{p} \sum_{a>p^{u}}^{p} \frac{1}{a} \pi_{q} / \pi_{p} \sum_{b>q^{u}}^{p, q} \frac{1}{b} \psi\left(\frac{x}{a b p q}, q\right)=S_{1}+S_{2} \text { say }
$$

where

$$
S_{1}=2 x \sum_{p<q \leqslant x} \frac{1}{p q} \pi_{p} \sum_{a>p^{u}}^{p} \frac{1}{a} \pi_{q} \mid \pi_{p} \sum_{b>q^{u}}^{p, q} \frac{1}{b}
$$

and

$$
S_{\mathbf{2}}=2 x \sum_{p<q \leqslant x} \frac{1}{p q} \pi_{p} \sum_{a>p^{u}}^{p} \frac{1}{a} \pi_{q} / \pi_{p} \sum_{b>q^{u}}^{p, q} \frac{1}{b}\left(\psi\left(\frac{\infty}{a b p q}\right)-1\right) .
$$

By (15) we can replace $\pi_{q} / \pi_{p} \sum_{b>q^{u}}^{p, q} \frac{1}{b}$ in $S_{1}$ by $\tau(u)$ and we get

$$
\begin{aligned}
S_{1} & =2 x \tau(u) \sum_{p<q \leqslant x} \frac{1}{p q} \pi_{p} \sum_{a>p^{u}}^{p} \frac{1}{a}+E \\
& =2 x(\tau(u))^{2} \sum_{p<q \leqslant x} \frac{1}{p q}+O\left(2 x \tau(u) \sum_{p<q \leqslant x}\left(\frac{\log _{2} p}{q p \log p}\right)\right)+E \\
& =x\left(\tau(u) \log _{2} x\right)^{2}+E,
\end{aligned}
$$

which gives us the main term.

$\mathrm{S}_{2}$ can be dealt with in the same way as its counterpart in Theorem 1. We write

$$
x^{\prime}=x^{1 /\left(2 u+2+3 \log _{2} x\right)}
$$

and divide the sum up as follows:
(i) $x^{\prime}<q \leqslant x$,
(ii) $a>p^{u+\log _{2} x}$ or $b>q^{u+\log _{2} x}$
(iii) $q<\mathfrak{x}^{\prime}, p^{u}<a \leqslant p^{u+\log _{2} x}, q^{u}<b \leqslant q^{u+\log _{2} x}$.
We then find that $S_{2}=x o\left(\left(\tau(u) \log _{2} x\right)^{2}\right)+o(x)$ and this completes the proof of (13).

Theorem 1 (ii) now follows easily.

$$
\begin{aligned}
& \sum_{n \leqslant x}\left(\varphi(u, n)-\tau(u) \log _{2} n\right)^{2} \\
& =\sum_{n \leqslant x} \varphi(u, n)^{2}-2 \tau(u) \sum_{n \leqslant x} \varphi(u, n) \log _{2} n+\tau(u)^{2} \sum_{n \leqslant x}\left(\log _{2} n\right)^{2} \\
& =x\left\{o\left(\left(\tau(u) \log _{2} x\right)^{2}\right)+O\left(\tau(u) \log _{2} x\right)+O(1)\right\}+ \\
& \quad+2 \tau(u) \sum_{n \leqslant x} \varphi(u, n)\left(\log _{2} x-\log _{2} n\right)
\end{aligned}
$$

but the sum on the right is easily shown to be $o(x)$ and the result follows. 
[1] N. G. de Bruijn, On the number of uncancelled elements in the sieve of Err sthenes, Indag. Math. 12 (1950), pp. 247-256.

[2] - On the number of positive integers $\leqslant x$ and free of prime factors $>y$, ibid. (1951), pp. 50-60.

[3] - The asymptotic behaviour of a function occurring in the theory of primes, J. Ind: Math. Soc. (N.S.) 15 (195I), pp. 25-32.

[4] P. Erdös, On some properties of prime factors of integers, Nagoya Math. J. (1966), pp. 617-623.

[5] C. G. Esseen, Fourier analysis of distribution functions. A mathematical sth of the Laplace-Gaussian law, Acta Math. 77 (1945), pp. 1-125.

On character sums and the non-vanishing for $s>0$ of Dirichlet $L$-series belonging to real odd characters $\chi$

by

S. Ghowla (Princeton, N. J.), I. Kessler, and M. Lutvingston (Edwardsville, Tll.)

1. Introduction. Let $\chi$ be a real non-principal character $\bmod k$. If

$$
\sum_{n=1}^{\infty} \chi(n) \geqslant 0 \quad \text { for all } x
$$

it follows by partial summation that

(1.2) $L(s, \chi)=\sum_{n=1}^{\infty} \frac{\chi(n)}{n^{s}}$ has no real zeros in the interval $0<s<1$,

and

(1.3) $\quad L(1, \chi)>c$ where $c$ is some positive absolute constant $>2 / 3$.

At the present time it is not known if there are infinitely many real primitive characters $x$ for which (1.2) holds. On the other hand, it has been shown that if $\chi$ is a real primitive character mod $k$ then $\varlimsup_{k \rightarrow \infty} \frac{L(1, \chi)}{\log \log k}$ $>0$ ([2], [8]), but it is unknown if the $k$ 's for which (1.3) holds have a nonzero density in the sequence of positive integers.

The results of our numerical investigations concerning the primes $p \equiv 3(\bmod 4)$ for which (1.1) holds suggest that these primes possess a positive limiting frequency in the sequence of all rational primes $\equiv 3$ $(\bmod 4)$. Our results in this connection are presented in Section 2 of this paper. In the third section we have given a brief account of related recent work and open problems on character sums. The final section consists of tables displaying pertinent computational results.

2. In this section we assume $\chi$ is a real primitive character mod $k$, where $k$ is prime, and thus we may take $\chi(n)$ to be the Legendre symbol $\left(\frac{n}{k}\right)$.

6 - Acta Arithmetica $\mathrm{XXXIII,1}$ 